\title{
A note on the Miller-Tucker-Zemlin model for the asymmetric traveling salesman problem
}

\author{
T. SAWIK* \\ AGH University of Science and Technology, Department of Operations Research and Information Technology, \\ 30 Mickiewicza Av., 30-059 Kraków, Poland
}

\begin{abstract}
An enhancement of the Miller-Tucker-Zemlin (MTZ) model for the asymmetric traveling salesman problem is presented by introducing additional constraints to the initial formulation. The constraints account for ordering of boundary nodes as well as all successive nodes in the salesman tour. The enhanced MTZ subtour elimination constraints are computationally compared with the basic MTZ constraints and the version of MTZ lifted by Desrochers and Laporte. The proposed enhancement shows improved performance on a number of asymmetric TSPLIB instances.
\end{abstract}

Key words: asymmetric traveling salesman problem, Miller-Tucker-Zemlin constraints, subtour elimination constraints.

\section{Introduction}

The asymmetric traveling salesman problem (ATSP) is defined on a directed network in which travels are allowed only in the direction specified. Given a network of $n$ nodes $i \in V=\{1, \ldots, n\}$, set $A \subset V \times V$ of directed arcs between the nodes and the distance $c_{i j}$ associated with each $\operatorname{arc}(i, j) \in A$. A salesman who begins trip in node 1 , which is the depot, must visit each node exactly once and return to the node 1 . The problem is to find the shortest directed tour for visiting $n$ nodes. The basic formulation for the ATSP problem is as follows (see e.g. [1]).

Minimize

subject to

$$
\sum_{(i, j) \in A} c_{i j} x_{i j}
$$

do not contain subtours;

$$
\begin{gathered}
\sum_{i:(i, j) \in A} x_{i j}=1, j \in V, \\
\sum_{j:(i, j) \in A} x_{i j}=1, i \in V, \\
\left\{(i, j) \in A: x_{i j}=1\right\}
\end{gathered}
$$

$$
x_{i j} \in\{0,1\} ;(i, j) \in A,
$$

where $x_{i j}=1$, if directed arc $(i, j) \in A$ is in the tour; otherwise $x_{i j}=0$.

Constraints (4) eliminate subtours not containing the depot node 1 . The subtour elimination condition (4) can be formulated in different ways. One of the most efficient approach derives from the paper [2] of Dantzig, Fulkerson, and Johnson

$\sum_{i \in S} \sum_{j \in S} x_{i j} \leq|S|-1 ; \quad \forall S \subset\{2, \ldots, n\},|S| \geq 2$,

\footnotetext{
*e-mail: ghsawik@cyf-kr.edu.pl
}

and implies that the number of arcs which can be packed in the clique defined by the set of nodes $S$ cannot exceed $|S|-1$. Formula (6) introduces $O\left(2^{n}\right)$ constraints and defines facets of the ATSP polytope. A practical importance owns two-node version of (6), namely

$$
x_{i j}+x_{j i} \leq 1 ; i, j \in V: i \neq j .
$$

Inequality (7) means that the tour may contain either arc $(i, j)$, $\left(x_{i j}=1\right)$, or arc $(j, i),\left(x_{j i}=1\right)$ or neither of these two arcs, $\left(x_{i j}=0\right.$ and $\left.x_{j i}=0\right)$.

Another most-known tractable formulation of subtour elimination constraints (MTZ) follows from Miller, Tucker, and Zemlin [3] and has the form of

$$
u_{i}-u_{j}+n x_{i j} \leq n-1 ; i, j \in\{2, \ldots n\}, i \neq j
$$

$$
u_{i} \in \mathcal{R} ; i \in V: i>1 \text {, }
$$

where the additional real variables $u_{i}$ are used to give an ordering to all nodes excluding the depot to prevent the formation of illegal subtours. The $u_{i}$ variables are unrestricted in the original paper [3]. Since the tour begins in the depot node $i=1$, (9) can be replaced by the following constraints (10), (11), that do not affect the LP bound of the ATSP

$$
\begin{gathered}
u_{1}=1 \\
2 \leq u_{i} \leq n ; i \in V: i>1
\end{gathered}
$$

where $u_{i}$ denotes the position of node $i$ in the tour.

The polynomial in number $\left(O\left(n^{2}\right)\right)$, MTZ constraints are known to produce a weak LP relaxation of the ATSP. However, they are flexible and capable of solving small to medium sized problems to optimality, using commercially available optimizers for mixed integer programming (MIP). The commercially available solvers focus on identifying efficient cutting planes 
during branch-and-bound-and-cut process, to successively reduce the size of the feasible polyhedral region. On the other hand the researchers seek to tighten the polyhedral representation of the initial ATSP formulation to use the best bounds produced by the linear programming relaxation of the initial formulation that guides branching decisions, regardless of the run-time actions taken by the MIP optimizers. There exists an extensive literature on the MTZ constraints, e.g., [4-8]. For a recent overview, see [9].

In this note a simple enhancement of the MTZ constraints is presented, The constraints account for ordering of boundary nodes, immediate neighbours of the depot node, as well as all intermediate nodes in the salesman tour. The boundary constraints restrict the selection of the first and the last arc of the salesman tour, while the intermediate constraints restrict the selection of all intermediate arcs. The intermediate constraints include the basic MTZ subtour elimination constraints. The proposed enhancement of the MTZ formulation leads to an improved performance of the MTZ-based subtour elimination constraints, which will be illustrated with a set of computational examples.

\section{Enhancement of the MTZ formulation}

The MTZ formulation for the ATSP (minimize (1) subject to $(2,3,5,7,8,10,11))$ is enhanced by the addition of new constraints derived in this section. The constraints account for ordering of boundary nodes as well as all intermediate nodes in the salesman tour.

2.1. Boundary conditions. First, the boundary conditions are formulated for the immediate successor and immediate predecessor of the depot node.

If node $j>1$ is the first one in the tour, i.e., the salesman travels from the depot node 1 directly to node $j,\left(x_{1 j}=1\right)$, then the position of node $j$ in the tour is $u_{j}=u_{1}+1$. Thus, if $x_{1 j}=1$ then $u_{j}=u_{1}+1$. This condition can be modeled with the following inequality

$$
u_{j}-u_{1}+(n-2) x_{1 j} \leq n-1 ; j \in V: j>1
$$

If $x_{1 j}=1$ then Eqs. (10-12) imply $u_{j}=2$; otherwise Eq. (12) implies $u_{j} \leq n$, and hence is inactive.

If node $i>1$ is the last one in the tour, i.e., the salesman returns from node $i$ directly to the depot node $1,\left(x_{i 1}=1\right)$, then the position of node $i$ in the tour is $u_{i}=u_{1}+n-1$. Thus, if $x_{i 1}=1$ then $u_{i}=u_{1}+n-1$. This condition can be modelled with the following inequality

$$
u_{1}-u_{i}+(n-1) x_{i 1} \leq 0 ; i \in V: i>1
$$

If $x_{i 1}=1$ then Eqs. $(10,11)$ and $(13)$ imply $u_{i}=n$; otherwise Eq. (13) implies $u_{i} \geq 1$, and hence is inactive.

Equations (12) and (13) define the boundary conditions for the salesman tour.
2.2. Intermediate conditions. The intermediate conditions for any pair of successive nodes in the tour are formulated below.

If the salesman travels from node $i$ directly to node $j,\left(x_{i j}=1\right.$ and $\left.x_{j i}=0\right)$ then $u_{j}=u_{i}+1$. If neither $\operatorname{arc}(i, j)$ or $\operatorname{arc}(j, i)$ are in the tour $\left(x_{i j}=0\right.$ and $\left.x_{j i}=0\right)$ then $\left|u_{j}-u_{i}\right| \leq n-1$ or equivalently $1-n \leq u_{j}-u_{i} \leq n-1$. The above conditions can be modelled with the following inequalities

$$
\begin{gathered}
1-n+n x_{i j} \leq u_{j}-u_{i} \leq n-1-(n-2) x_{i j} \\
i, j \in V: i \neq j, i>1, j>1 .
\end{gathered}
$$

If $x_{i j}=1$ then Eq. (14) is converted to $1 \leq u_{j}-u_{i} \leq 1 ; i, j \in V$ : $i \neq j, i>1, j>1$, which implies $u_{j}-u_{i}=1 ; i, j \in V$ : $i \neq j, i>1, j>1$. Otherwise $\left|u_{j}-u_{i}\right| \leq n-1 ; i, j \in V$ : $i \neq j, i>1, j>1$ and hence Eq. (14) is inactive.

The two inequalities of Eq. (14) can be rewritten separately as below

$$
\begin{gathered}
u_{i}-u_{j}+n x_{j i} \leq n-1 ; i, j \in V: i \neq j, j>1 \\
u_{i}-u_{j}+(n-2) x_{j i} \leq n-1 \\
i, j \in V: i \neq j, i>1 .
\end{gathered}
$$

Equations (15) and (16) define the intermediate conditions for the salesman tour, where inequalities (15) are the Miller-Tucker-Zemlin subtour elimination constraints (8), and Eq. (16) is the right-hand side inequality of (14) for the reversed pair $(j, i)$. Conditions (16) can be considered complementary to MTZ conditions, (15). Note that Eq. (16) for $j=1$ and Eq. (12) are redundant.

The inequalities (15) and (16) can be considered a "disaggregation" of the lifted version of the MTZ constraints [4], for the node ordering variables, $u_{i}$, defined in Eqs. $(10,11)$ :

$$
\begin{gathered}
u_{i}-u_{j}+n x_{i j}+(n-2) x_{j i} \leq n-1 \\
i, j \in V: i \neq j, i>1, j>1 .
\end{gathered}
$$

The lifted MTZ constraints (17), which are considered to be one of the most efficient improvements (e.g., [9]), will be computationally compared with the proposed enhanced MTZ constraints.

Denote by ATSP_MTZen, the formulation with the enhanced MTZ constraints $(12,13,15,16)$,

ATSP_MTZen: Minimize (1) subject to $(2,3,5,7,10$, $11,12,13,15,16)$

by ATSP MTZen-, the formulation ATSP MTZen without Eqs. (10) and (12),

ATSP MTZen-: Minimize (1) subject to $(2,3,5,7,11$, $13,15,16)$,

by ATSP_MTZ, the formulation with the basic MTZ constraints (15),

ATSP MTZ: Minimize (1) subject to $(2,3,5,7,10$, $11,15)$,

and by ATSP DL, the formulation with the lifted MTZ constraints (17) and without Eq. (10),

ATSP_DL: Minimize (1) subject to $(2,3,5,7,11,17)$. 
Note that removing of Eq. (10) leads to the ATSP models with a greater number of active constraints, which may result in tighter MIP formulations.

The performance of the above four ATSP formulations will be compared on a set of test instances.

\section{Computational examples}

The computational experiments were performed using the AMPL programming language and the CPLEX 12.6.2 solver with the default setting, on a MacBookPro laptop with Intel Core i7 processor running at $2.8 \mathrm{GHz}$ and with $16 \mathrm{~GB}$ RAM. Table 1 presents comparison of computational results on a number of asymmetric TSPLIB [10] instances with the number of nodes ranging from 17 to 443. Of the two enhanced models ATSP_MTZen and ATSP_MTZen-, the results of that requiring smaller CPU time are presented only. For each of the ATSP formulations, the table presents optimal solution value Opt., (1), solution value of the LP relaxation, CPU time in seconds required to find proven optimal solution or GAP\% after $3600 \mathrm{CPU}$ seconds, and the size of the ATSP model after presolving: number of variables, Var., number of binary variables, Bin., and number of constraints, Cons. The models have identical number of variables and differ in the number of constraints, which is largest for ATSP MTZen-, and identical for ATSP_MTZ and ATSP_DL models.

The LP relaxation of the ATSP models is obtained by replacing constraints (5) by $0 \leq x_{i j} \leq 1 ; \forall(i, j) \in A$. In most cases, the LP relaxation values are identical for all models, which indicates that the polyhedral representation of the initial ATSP formulation has not been tightened by the proposed MTZ enhancement.

Model ATSP_MTZen- with the enhanced MTZ constraints was capable of finding proven optimal solution for all test instances within 3600 CPU seconds, while models ATSP MTZ and ATSP_DL failed to prove optimality for the most challenging problem p43.atsp. Neither ATSP_MTZ nor ATSP_DL were able to close the LP-IP gap within the preset CPU time limit. In addition, model ATSP_DL failed to find any feasible solution for rbg358.atsp using the CPLEX solver with the default setting. A custom setting or using a different MIP optimizer might overcome the above difficulties.

Table 1

Comparison of ATSP_MTZen (or ATSP_MTZen-), ATSP_MTZ and ATSP_DL formulations

\begin{tabular}{|c|c|c|c|c|c|c|c|c|c|c|c|}
\hline Instance & $n$ & Opt. & \multicolumn{3}{|c|}{ Model ATSP_MTZen (or ATSP_MTZen-) } & \multicolumn{3}{|c|}{$\begin{array}{c}\text { Model ATSP_MTZ } \\
\text { CPU[sec] }\end{array}$} & \multicolumn{3}{|c|}{$\begin{array}{c}\text { Model ATSP_DL } \\
\text { CPU[sec] }\end{array}$} \\
\hline br17.atsp & 17 & 39 & 22 & $<1$ & $288,272,818$ & 22 & $<1$ & 546 & 22 & $<1$ & 546 \\
\hline ftv33.atsp & 34 & 1286 & 1215 & $<1$ & $1155,1122,3368$ & 1215 & $<1$ & 2246 & 1217 & $<1$ & 2246 \\
\hline ftv35.atsp & 36 & 1473 & 1413 & $<1$ & $1295,1260,3782$ & 1413 & $<1$ & 2522 & 1413 & $<1$ & 2522 \\
\hline ftv38.atsp & 39 & 1530 & 1476 & $<1$ & $1520,1582,4482$ & 1476 & $<1$ & 2966 & 1476 & $<1$ & 2966 \\
\hline p43.atsp & 43 & 5620 & 216 & $967^{(-)}$ & $1849,1806,5504$ & 216 & $0.14 \%$ & 3616 & 216 & $0.11 \%$ & 3616 \\
\hline ftv47.atsp & 48 & 1776 & 1725 & 3 & $2303,2256,6770$ & 1725 & 3 & 4514 & 1725 & 2 & 4514 \\
\hline ry $48 p . a t s p$ & 48 & 14422 & 13809 & 20 & $2303,2256,6770$ & 13809 & 10 & 4514 & 13809 & 18 & 4514 \\
\hline $\mathrm{ft} 53$.atsp & 53 & 6905 & 6011 & 18 & $2808,2756,8270$ & 6010 & 19 & 5514 & 6011 & 23 & 5514 \\
\hline $\mathrm{ft} 55$.atsp & 56 & 1608 & 1511 & 4 & $3135,3080,9242$ & 1510 & 1 & 6162 & 1511 & 3 & 6162 \\
\hline ft70.atsp & 70 & 38673 & 38333 & 9 & $4899,4830,14492$ & 38325 & 5 & 9662 & 38326 & 4 & 9662 \\
\hline ftv70.atsp & 71 & 1950 & 1859 & 12 & $5040,4970,14912$ & 1858 & 7 & 9942 & 1859 & 7 & 9942 \\
\hline kro124p.atsp & 100 & 36230 & 34974 & 140 & $10000,9900,29801$ & 34977 & 77 & 19802 & 34977 & 77 & 19802 \\
\hline ftv170.atsp & 171 & 2755 & 2698 & 45 & $29240,29070,87212$ & 2698 & 195 & 58412 & 2698 & 460 & 58142 \\
\hline rbg323.atsp & 323 & 1326 & 1326 & 59 & $104328,104006,312020$ & 1326 & 428 & 208014 & 1326 & 434 & 208014 \\
\hline rbg358.atsp & 358 & 1163 & 1163 & $173^{(-)}$ & $128164,127806,384134$ & 1163 & 579 & 255614 & 1163 & $\dagger$ & 255614 \\
\hline rbg403.atsp & 403 & 2465 & 2465 & 187 & $162408,162006,486020$ & 2465 & 503 & 324014 & 2465 & 31 & 324014 \\
\hline rbg443.atsp & 443 & 2720 & 2720 & 1885 & $196248,195806,587420$ & 2720 & 1735 & 391614 & 2720 & 1129 & 391614 \\
\hline
\end{tabular}

$(-)$ - CPU seconds for model ATSP MTZen-

Opt. - optimal solution value of (1), LP $=$ LP relaxation solution value of (1)

Var. - number of variables, Bin. - number of binary variables, Cons. - number of constraints $\mathrm{CPU}[\mathrm{sec}] / \mathrm{GAP} \%$ - CPU seconds for proven optimal solution or GAP\% after $3600 \mathrm{CPU}$ seconds $\dagger-$ no feasible solution within 3600 CPU seconds 
Except for the smallest size problems, where CPU time required finding proven optimal solutions was less than one second for all models, the performance of the four ATSP models was mixed. However, the enhanced MTZ formulation outperforms the other two models on the most challenging problem instances.

\section{Conclusions}

In this paper an enhancement of the MTZ subtour elimination constraints is presented by the addition of more constraints to the initial formulation. The constraints account for ordering of boundary nodes, immediate neighbours of the depot node, as well as all intermediate nodes in the salesman tour. The boundary constraints restrict the selection of the first and the last arc of the salesman tour, while the intermediate constraints restrict the selection of all intermediate arcs. The intermediate constraints include the basic MTZ subtour elimination constraints.

The computational results indicate that the proposed MTZ enhancement does not tighten the polyhedral representation of the initial ATSP formulation to obtain better bounds produced by the linear programming relaxation of the initial formulation. However, the computational results clearly demonstrate that the enhancement of the MTZ formulation by introducing additional constraints to the initial formulation may improve performance of subtour elimination constraints for the asymmetric traveling salesman problem and shorten CPU time required to find proven optimal solutions. The further research should concentrate on identifying special data structure of the ATSP and deriving the corresponding cuts that can be added to the initial formulations.

While the purpose of this paper was to enhance an exact ATSP formulation capable of solving small to medium sized problems to optimality using commercially available optimizers for MIP, there is a large body of literature on heuristics for large sized problems, e.g. [11]. There are three general classes of such heuristics: classical tour construction heuristics such as the greedy-type algorithms, e.g. [12], local search algorithms based on re-arranging segments of the tour, such as the Kanellakis-Papadimitriou algorithm [13], and algorithms based on patching together the cycles in a minimum cycle cover, such as Zhang algorithm [14].

Acknowledgments. The author is grateful to two anonymous reviewers for very careful reading of manuscript and providing comments which helped to improve this paper. This work has been supported by NCN research grant DEC-2013/11/B/ ST8/04458, and by AGH\#11/11.200.

\section{REFERENCES}

[1] T.Öncan, I.K. Altinel and G. Laporte, "A comparative analysis of several asymmetric traveling salesman problem formulations", Computers \& Operations Research 36, 637-654 (2009).

[2] G.B. Dantzig, D.R. Fulkerson and S.M. Johnson, "Solution of a large-scale traveling salesman problem", Operations Research 2, 393-410 (1954).

[3] C.E. Miller, A.W. Tucker and R.A. Zemlin, "Integer programming formulations and traveling salesman problems", Journal of Association for Computing Machinery 7, 326-329 (1960).

[4] M. Desrochers and G. Laporte, "Improvements and extensions to the Miller-Tucker-Zemlin subtour elimination constraints", Operations Research Letters 10, 27-36 (1991).

[5] L. Gouveia and J.M. Pires, "The asymmetric travelling salesman problem and a reformulation of the Miller-Tucker-Zemlin constraints", European Journal of Operational Research 112, 134146 (1999).

[6] L. Gouveia and J.M. Pires, "The asymmetric travelling salesman problem: On generalizations of disaggregated Miller-Tucker-Zemlin constraints", Discrete Applied Mathematics 112, 129-145 (2001).

[7] H.D. Sherali and P.J. Driscoll, "On tightening the relaxations of Miller-Tucker-Zemlin formulations for asymmetric travelling salesman problems", Operations Research 50(4), 656-669 (2002).

[8] S.C. Sarin, H.D. Sherali and A. Bhootra, "New tighter polynomial length formulations for the asymmetric traveling salesman problem with and without precedence constraints", Operations Research Letters 33, 62-70 (2005).

[9] T. Bektas and L. Gouveia, "Requiem for the Miller-Tucker-Zemlin subtour elimination constraints?", European Journal of Operational Research 236, 820-832 (2014).

[10] TSPLIB, "A library of travelling salesman and related problem instances", http://elib.zib.de/pub/mp-testdata/tsp/tsplib/tsplib. html (1997). Accessed 25.09.2015.

[11] J. Cirasella, D.S. Johnson, L.A. McGeoch and W. Zhang, "The Asymmetric Traveling Salesman Problem: Algorithms, Instance Generators, and Tests", in A. L. Buchsbaum and J. Snoeyink (eds.), Algorithm Engineering and Experimentation, Lecture Notes in Computer Science, 2153, 32-59 (2001).

[12] G. Gutin, A. Yeo and A. Zverovich, "Traveling salesman should not be greedy: domination analysis of greedy-type heuristics for the TSP", Discrete Applied Mathematics 117, 81-86 (2002).

[13] P. C. Kanellakis and C. H. Papadimitriou, "Local search for the asymmetric traveling salesman problem", Operations Research 28, 1086-1099 (1980).

[14] W. Zhang, "Truncated branch-and-bound: A case study on the asymmetric TSP", Proc. of AAAI 1993 Spring Symposium on AI and NP-Hard Problems, 160-166 (1993). 